\title{
BOUNDS FOR TAIL PROBABILITIES OF MARTINGALES USING SKEWNESS AND KURTOSIS
}

\author{
V. BentKus ${ }^{1}$ And T. JušKevičIus ${ }^{1}$
}

January 2008

\begin{abstract}
Let $M_{n}=X_{1}+\cdots+X_{n}$ be a sum of independent random variables such that $X_{k} \leq 1$, $\mathbb{E} X_{k}=0$ and $\mathbb{E} X_{k}^{2}=\sigma_{k}^{2}$ for all $k$. Hoeffding 1963, Theorem 3, proved that

$$
\mathbb{P}\left\{M_{n} \geq n t\right\} \leq H^{n}(t, p), \quad H(t, p)=(1+q t / p)^{p+q t}(1-t)^{q-q t}
$$

with

$$
q=\frac{1}{1+\sigma^{2}}, \quad p=1-q, \quad \sigma^{2}=\frac{\sigma_{1}^{2}+\cdots+\sigma_{n}^{2}}{n}, \quad 0<t<1 .
$$

Bentkus 2004 improved Hoeffding's inequalities using binomial tails as upper bounds. Let $\gamma_{k}=$ $\mathbb{E} X_{k}^{3} / \sigma_{k}^{3}$ and $\varkappa_{k}=\mathbb{E} X_{k}^{4} / \sigma_{k}^{4}$ stand for the skewness and kurtosis of $X_{k}$. In this paper we prove (improved) counterparts of the Hoeffding inequality replacing $\sigma^{2}$ by certain functions of $\gamma_{1}, \ldots, \gamma_{n}$ respectively $\varkappa_{1}, \ldots, \varkappa_{n}$. Our bounds extend to a general setting where $X_{k}$ are martingale differences, and they can combine the knowledge of skewness and/or kurtosis and/or variances of $X_{k}$. Up to factors bounded by $e^{2} / 2$ the bounds are final. All our results are new since no inequalities incorporating skewness or kurtosis control so far are known.
\end{abstract}

\section{Introduction AND RESULts}

In a celebrated paper of Hoeffding 1963 several inequalities for sums of bounded random variables were established. For improvements of the Hoeffding inequalities and related results see, for example, Talagrand 1995, McDiarmid 1989, Godbole and Hitczenko 1998, Pinelis 19982007, Laib 1999, B 2001-2007, van de Geer 2002, Perron 2003, BGZ 2006-2006, BGPZ 2006, BKZ 2006, 2007, BZ 2003, etc. Up to certain constant factors, these improvements are close to the final optimal inequalities, see B 2004, BKZ 2006. However so far no bounds taking into account information related to skewness and/or kurtosis are known, not to mention certain results related to symmetric random variables, see BGZ 2006, BGPZ 2006. In this paper we prove general and optimal counterparts of Hoeffding's 1963 Theorem 3, using assumptions related to skewness and/or kurtosis.

\footnotetext{
${ }^{1}$ Vilnius Institute of Mathematics and Informatics. Supported by grant ???

1991 Mathematics Subject Classification. 60E15.

Key words and phrases. skewness, kurtosis, Hoeffding's inequalities, sums of independent random variables, martingales, bounds for tail probabilities, Bernoulli random variables, binomial tails, probabilities of large deviations, method of bounded differences.
} 
Let us recall Hoeffding's 1963 Theorem 3. Let $M_{n}=X_{1}+\cdots+X_{n}$ be a sum of independent random variables such that $X_{k} \leq 1, \mathbb{E} X_{k}=0$, and $\mathbb{E} X_{k}^{2}=\sigma_{k}^{2}$ for all $k$. Write

$$
\sigma^{2}=\frac{\sigma_{1}^{2}+\cdots+\sigma_{n}^{2}}{n}, \quad p=\frac{\sigma^{2}}{1+\sigma^{2}}, \quad q=1-p .
$$

Hoeffding 1963, Theorem 3, established the inequality

$$
\mathbb{P}\left\{M_{n} \geq n t\right\} \leq H^{n}(t, p), \quad H(t, p)=(1+q t / p)^{p+q t}(1-t)^{q-q t}
$$

assuming that $0<t<1$. One can rewrite $H^{n}(x, p)$ as

$$
H^{n}(x, p)=\inf _{h>0} \exp \{-h n t\} \mathbb{E} \exp \left\{h T_{n}\right\},
$$

where $T_{n}=\varepsilon_{1}+\cdots+\varepsilon_{n}$ is a sum of $n$ independent copies of a Bernoulli random variable, say $\varepsilon=\varepsilon\left(\sigma^{2}\right)$, such that

$$
\mathbb{P}\left\{\varepsilon=-\sigma^{2}\right\}=q, \quad \mathbb{P}\{\varepsilon=1\}=p, \quad \mathbb{E} \varepsilon^{2}=\sigma^{2} .
$$

Using the shorthand $x=n t$, we can rewrite the Hoeffding result as

$$
\mathbb{P}\left\{M_{n} \geq x\right\} \leq \inf _{h>0} \mathrm{e}^{-h x} \mathbb{E} \mathrm{e}^{h T_{n}},
$$

In B 2004 the inequality (1.3) is improved to

$$
\mathbb{P}\left\{M_{n} \geq x\right\} \leq \inf _{h<x}(x-h)^{-2} \mathbb{E}\left(T_{n}-h\right)_{+}^{2} .
$$

Actually, inequalities (1.1), (1.3) and (1.4) extend to cases where $M_{n}$ is a martingale or even super-martingale, see B 2004 for a proof. In the case of (1.1) and (1.3) this was noted already by Hoeffding 1963.

The right hand side of (1.4) satisfies

$$
\inf _{h<x}(t-h)^{-2} \mathbb{E}\left(T_{n}-h\right)_{+}^{2} \leq \frac{\mathrm{e}^{2}}{2} \mathbb{P}\left\{T_{n} \geq x\right\}, \quad \mathrm{e}=2.718 \ldots
$$

for integer $x \in \mathbb{Z}$. For non-integer $x$ one has to interpolate the probability log-linearly, see B 2004 for details. The right-hand side of (1.4) can be given explicitly as a function of $x, p$ and $n$, see BKZ 2006, as well as Section 2 of the present paper. To have bounds as tight as possible is essential for statistical applications, like those in audit, see BZ 2003.

Our intention in this paper is to develop methods leading to counterparts of (1.1), (1.3) and (1.4) such that information related to the skewness and kurtosis

$$
\gamma_{k}=\frac{\mathbb{E}\left(X_{k}-\mathbb{E} X_{k}\right)^{3}}{\sigma_{k}^{3}}, \quad \varkappa_{k}=\frac{\mathbb{E}\left(X_{k}-\mathbb{E} X_{k}\right)^{4}}{\sigma_{k}^{4}}
$$

of $X_{k}$ is taken into account (in this paper we define $\gamma_{k}=\infty$ and $\varkappa_{k}=1$ if $\sigma_{k}=0$ ). All our results hold in general martingale setting. 
All known proofs of inequalities of type (1.3) and (1.4) start with an application of Chebyshev's inequality. For example, in the case of (1.4) we can estimate

$$
\mathbb{P}\left\{M_{n} \geq x\right\} \leq \inf _{h<x}(x-h)^{-2} \mathbb{E}\left(M_{n}-h\right)_{+}^{2}
$$

since the indicator function $t \mapsto \mathbb{I}\{t \geq x\}$ obviously satisfies $\mathbb{I}\{t \geq x\} \leq(x-h)^{-2}(t-h)_{+}^{2}$ for all $t \in \mathbb{R}$. The further proof of (1.4) consists in showing that $\mathbb{E}\left(M_{n}-h\right)_{+}^{2} \leq \mathbb{E}\left(T_{n}-h\right)_{+}^{2}$ for all $h \in \mathbb{R}$. We would like to emphasize that all our proofs are optimal in the sense that no further improvements are possible in estimation of $\mathbb{E}\left(M_{n}-h\right)_{+}^{2}$. Indeed, in the special case $M_{n}=T_{n}$ the inequality $\mathbb{E}\left(M_{n}-h\right)_{+}^{2} \leq \mathbb{E}\left(T_{n}-h\right)_{+}^{2}$ turns into the equality $\mathbb{E}\left(T_{n}-h\right)_{+}^{2}=\mathbb{E}\left(T_{n}-h\right)_{+}^{2}$.

In view of (1.7) it is natural to introduce and to study transforms $G \mapsto G_{\beta}$ of survival functions $G(x)=\mathbb{P}\{X \geq x\}$ of the type

$$
G_{\beta}(x)=\inf _{h<x}(x-h)^{-\beta} \mathbb{E}(X-h)_{+}^{\beta}, \quad \beta>0,
$$

defining $G_{0}=G$ in the case $\beta=0$. See Pinelis 1988, 1989, B 2004, BKZ 2006 for related known results.

The paper is organized as follows. In the Introduction we provide necessary definitions and formulations of our results, including their versions for sums of martingale differences. In Section 2 we recall a description of the transform $G \mapsto G_{2}$ of binomial survival functions - our bounds are given using $G_{2}$. Section 3 contains proofs of the results.

Henceforth $M_{n}=X_{1}+\cdots+X_{n}$ stands for a martingale sequence such that the differences $X_{k}$ are uniformly bounded (we set $M_{0}=X_{0}=0$ ). Without loss of generality we can assume that the bounding constant is 1 , that is, that $X_{k} \leq 1$. Let $\mathcal{F}_{0} \subset \mathcal{F}_{1} \subset \cdots \subset \mathcal{F}_{n}$ be a related sequence of $\sigma$-algebras such that $M_{k}$ are $\mathcal{F}_{k}$-measurable. Introduce the conditional variance $s_{k}^{2}$, skewness $g_{k}$ and kurtosis $c_{k}$ of $X_{k}$ by

$$
s_{k}^{2}=\mathbb{E}\left(X_{k}^{2} \mid \mathcal{F}_{k-1}\right), \quad g_{k}=\mathbb{E}\left(X_{k}^{3} \mid \mathcal{F}_{k-1}\right) / s_{k}^{3}, \quad c_{k}=\mathbb{E}\left(X_{k}^{4} \mid \mathcal{F}_{k-1}\right) / s_{k}^{4} .
$$

Note that $s_{k}^{2}, g_{k}, c_{k}$ are $\mathcal{F}_{k-1}$-measurable random variables.

Remark 1.1. We prove our results using (1.4) for martingales. It is proved in B 2004 that all three inequalities (1.1), (1.3) and (1.4) hold with $\sigma^{2}=\left(\sigma_{1}^{2}+\cdots+\sigma_{n}^{2}\right) / n$ if $M_{n}$ is a martingale with differences $X_{k} \leq 1$ such that the conditional variances $s_{k}^{2}$ satisfy $s_{k}^{2} \leq \sigma_{k}^{2}$ for all $k$.

It is easy to check that Bernoulli random variables $\varepsilon=\varepsilon\left(\sigma^{2}\right)$ of type (1.2) have variance $\sigma^{2}$ and skewness $\gamma$ related as

$$
\gamma=\sigma-\frac{1}{\sigma}, \quad \sigma^{2}=u^{2}(\gamma), \quad \text { where } u(x)=\sqrt{1+\frac{x^{2}}{4}}-\frac{x}{2}
$$


Theorem 1.2. Assume that the differences $X_{k}$ of a martingale $M_{n}$ satisfy $X_{k} \leq 1$, and that the conditional skewness $g_{k}$ of $X_{k}$ are bounded from below by some non-random $\gamma_{k}$, that is, that

$$
g_{k} \geq \gamma_{k}, \quad k=1,2, \ldots, n .
$$

Then (1.3) and (1.4) hold with $T_{n}$ being a sum of $n$ independent copies of a Bernoulli random variable $\varepsilon=\varepsilon\left(\sigma^{2}\right)$ of type (1.2) with skewness $\gamma$ and variance $\sigma^{2}$ defined by

$$
\gamma=\frac{1}{\sqrt{n}} \frac{\gamma_{1} u\left(\gamma_{1}\right)+\cdots+\gamma_{n} u\left(\gamma_{n}\right)}{\sqrt{u^{2}\left(\gamma_{1}\right)+\cdots+u^{2}\left(\gamma_{n}\right)}}, \quad \sigma^{2}=\frac{u^{2}\left(\gamma_{1}\right)+\cdots+u^{2}\left(\gamma_{n}\right)}{n} .
$$

In the special case where all $\gamma_{k}$ are equal, $\gamma_{1}=\cdots=\gamma_{n}=\gamma$, the Bernoulli random variable has skewness $\gamma$ and variance $\sigma^{2}=u^{2}(\gamma)$.

It is easy to see that Bernoulli random variables $\varepsilon=\varepsilon\left(\sigma^{2}\right)$ of type (1.2) have variance $\sigma^{2}$ and kurtosis $\varkappa$ related as

$$
\varkappa=\frac{1}{\sigma^{2}}-1+\sigma^{2}, \quad 2 \sigma^{2}=\varkappa+1 \pm \sqrt{(\varkappa+1)^{2}-4} .
$$

In particular

$$
\sigma^{2} \leq v(\varkappa), \quad \text { where } 2 v(t)=t+1+\sqrt{(t+1)^{2}-4}
$$

Theorem 1.3. Assume that the differences $X_{k}$ of a martingale $M_{n}$ satisfy $X_{k} \leq 1$, and that the conditional kurtosis $c_{k}$ of $X_{k}$ are bounded from above by some non-random $\varkappa_{k}$, that is, that

$$
c_{k} \leq \varkappa_{k}, \quad k=1,2, \ldots, n .
$$

Then (1.3) and (1.4) hold with $T_{n}$ being a sum of $n$ independent copies of a Bernoulli random variable $\varepsilon=\varepsilon\left(\sigma^{2}\right)$ of type (1.2) with kurtosis $\varkappa$ and variance $\sigma^{2}$ defined by

$$
\varkappa=\frac{1}{\sigma^{2}}-1+\sigma^{2}, \quad \sigma^{2}=\frac{v\left(\varkappa_{1}\right)+\cdots+v\left(\varkappa_{n}\right)}{n},
$$

where the function $v$ is given in (1.14). In the special case where $\varkappa_{1}=\cdots=\varkappa_{n}=\varkappa$, the Bernoulli random variable has kurtosis $\varkappa$ and variance $\sigma^{2}=v(\varkappa)$.

The next Theorem 1.4 allows to combine our knowledge about variances, skewness and kurtosis. Theorems 1.2, 1.3 and (1.4), (1.4) for martingales (see Remark 1.1) are special cases of Theorem 1.4 setting in various combinations $\sigma_{k}^{2}=\infty, \gamma_{k}=-\infty, \varkappa_{k}=\infty$.

Theorem 1.4. Assume that the differences $X_{k}$ of a martingale $M_{n}$ satisfy $X_{k} \leq 1$, and that their conditional variances $s_{k}^{2}$, skewness $g_{k}$ and kurtosis $c_{k}$ satisfy

$$
s_{k}^{2} \leq \sigma_{k}^{2}, \quad g_{k} \geq \gamma_{k}, \quad c_{k} \leq \varkappa_{k}, \quad k=1,2, \ldots, n
$$

with some non-random $s_{k}^{2} \geq 0, g_{k} \geq-\infty$ and $1 \leq c_{k} \leq \infty$. Assume that numbers $\alpha_{k}^{2}$ satisfy

$$
\alpha_{k}^{2} \geq \min \left\{\sigma_{k}^{2}, u^{2}\left(\gamma_{k}\right), v\left(\varkappa_{k}\right)\right\}
$$


Then (1.3) and (1.4) hold with $T_{n}$ being a sum of $n$ independent copies of a Bernoulli random variable $\varepsilon=\varepsilon\left(\sigma^{2}\right)$ of type (1.2) with

$$
\sigma^{2}=\frac{\alpha_{1}^{2}+\cdots+\alpha_{n}^{2}}{n}
$$

where functions $u$ and $v$ are defined in (1.10) and (1.14) respectively.

Remark 1.5. All our inequalities can be extended to the case where $M_{n}$ is a supermartingale. Furthermore, their maximal versions hold, that is, in the left hand sides of these inequalities we can replace $\mathbb{P}\left\{M_{n} \geq x\right\}$ by $\mathbb{P}\left\{\max _{1 \leq k \leq n} M_{k} \geq x\right\}$.

Remark 1.6. One can estimate the right hand sides of our inequalities using Poisson distributions. In the case of Hoeffding's functions this is done by Hoeffding 1963. In notation of (1.1) his bound is

$$
H^{n}(t, p) \leq \inf _{h>0} \mathrm{e}^{-h x} \mathbb{E} \mathrm{e}^{h(\eta-\lambda)}=\exp \left\{x-(x+\lambda) \ln \frac{x+\lambda}{\lambda}\right\},
$$

where $x=t n, \lambda=n \sigma^{2}$, and $\eta$ is a Poisson random variable with parameter $\lambda$. It is shown in the proof of Theorem 1.1 in B 2004, that if $T_{n}$ is a sum of $n$ independent copies of a Bernoulli random variable $\varepsilon=\varepsilon\left(\sigma^{2}\right)$, then

$$
\inf _{h<x}(t-h)^{-2} \mathbb{E}\left(T_{n}-h\right)_{+}^{2} \leq \inf _{h<x}(t-h)^{-2} \mathbb{E}(\eta-\lambda-h)_{+}^{2},
$$

where $\eta$ is a Poisson random variable with parameter $\lambda=n \sigma^{2}$. The right hand side of (1.19) is given as an explicit function of $\lambda$ and $x$ in BKZ 2006.

Remark 1.7. A law of transformation $\left\{\sigma_{1}^{2}, \ldots, \sigma_{n}^{2}\right\} \mapsto \sigma^{2}$ in (1.1), (1.3) and (1.4) is a linear function. In bounds involving skewness and kurtosis corresponding transformations are nonlinear, see, for example, (1.12), where the transformation $\left\{\gamma_{1}, \ldots, \gamma_{n}\right\} \mapsto \gamma$ is given explicitly.

\section{An ANALYTIC DESCRIPTION OF TRANSFORMS $G_{2}$ OF BINOMIAL SURVIVAL FUNCTIONS $G$.}

In this section we recall an explicit analytical description of the right hand side of (1.4)

$$
G_{2}(x) \stackrel{\text { def }}{=} \inf _{h<x}(x-h)^{-2} \mathbb{E}\left(T_{n}-h\right)_{+}^{2},
$$

where $T_{n}$ is a sum of $n$ independent copies of the Bernoulli random variable (1.2). The description is taken from BKZ 2006. Let $G(x)=\mathbb{P}\left\{T_{n} \geq x\right\}$ be the survival function of $T_{n}$. The probabilities $p, q$ and the variance $\sigma^{2}$ are defined in (1.2). Write $\lambda=p n$. The sum $T_{n}=\varepsilon_{1}+\cdots+\varepsilon_{n}$ assumes the values

$$
d_{s}=-n \sigma^{2}+s\left(1+\sigma^{2}\right) \equiv \frac{s-\lambda}{q}, \quad s=0,1, \ldots, n
$$


The related probabilities satisfy

$$
p_{n, s}=\mathbb{P}\left\{T_{n}=d_{s}\right\}=\left(\begin{array}{c}
n \\
s
\end{array}\right) q^{n-s} p^{s} .
$$

The values $G\left(d_{s}\right)$ of the survival function of the random variable $T_{n}$ are given by

$$
G\left(d_{s}\right)=p_{n, s}+\cdots+p_{n, n}
$$

Write

$$
\nu_{n, s}=\frac{s p_{n, s}}{G\left(d_{s}\right)}
$$

Now we can describe the transform $G_{2}$. Consider a sequence

$$
0=r_{0}<r_{1}<\ldots<r_{n-1}<r_{n}=n
$$

of points which divide the interval $[0, n]$ into $n$ subintervals $\left[r_{s}, r_{s+1}\right]$. To define $G_{2}$ take

$$
r_{s}=\frac{\lambda-p \nu_{n, s}}{q \nu_{n, s}+\lambda-s}, \quad s=0,1, \ldots, n-1
$$

and

$$
G_{2}(x)=\frac{\lambda+\nu_{n, s}(s-\lambda-p)-q \nu_{n, s}^{2}}{q x^{2}-2 q \nu_{n, s}+\lambda+\nu_{n, s}(s-\lambda-p)} G\left(d_{s}\right), \quad r_{s} \leq x \leq r_{s+1} .
$$

\section{Proofs}

Proof of Theorem 1.2. This theorem is a special case of Theorem 1.4. Indeed, choosing

$$
\sigma_{k}^{2}=\infty, \quad \varkappa_{k}=\infty, \quad k=1,2, \ldots, n
$$

we have $v\left(\varkappa_{k}\right)=\infty$. Hence $\alpha_{k}^{2}$ from the condition of Theorem 1.4 have to satisfy $\alpha_{k}^{2} \geq u^{2}\left(\gamma_{k}\right)$. We choose $\alpha_{k}^{2}=u^{2}\left(\gamma_{k}\right)$. Then $\sigma^{2}=\left(u^{2}\left(\gamma_{1}\right)+\cdots+u^{2}\left(\gamma_{n}\right)\right) / n$. A small calculation shows that with such $\sigma^{2}$ the skewness $\gamma=\sigma-\frac{1}{\sigma}$ of Bernoulli random variables $\varepsilon=\varepsilon\left(\sigma^{2}\right)$ coincides with the expression given in (1.12)

Proof of Theorem 1.3. This theorem is a special case of Theorem 1.4. Indeed, choosing

$$
\sigma_{k}^{2}=\infty, \quad \gamma_{k}=-\infty, \quad k=1,2, \ldots, n,
$$

we have $u\left(\gamma_{k}\right)=\infty$. Hence $\alpha_{k}^{2}$ from the condition of Theorem 1.4 have to satisfy $\alpha_{k}^{2} \geq v\left(\varkappa_{k}\right)$. We choose $\alpha_{k}^{2}=v\left(\varkappa_{k}\right)$. Then $\sigma^{2}=\left(v\left(\varkappa_{1}\right)+\cdots+v\left(\varkappa_{n}\right)\right) / n$.

In the proof of Theorem 1.4 we use the next two lemmas. 
Lemma 3.1. Assume that a random variable $X \leq 1$ has mean $\mathbb{E} X=0$, variance $s^{2}=\mathbb{E} X^{2}$, and skewness such that $\frac{\mathbb{E} X^{3}}{s^{3}} \geq g$. Then

$$
s^{2} \leq u^{2}(g), \quad u(x) \stackrel{\text { def }}{=} \sqrt{1+\frac{x^{2}}{4}}-\frac{x}{2} .
$$

Proof. It is clear that

$$
\left(t+s^{2}\right)^{2}(1-t) \geq 0 \quad \text { for } t \leq 1
$$

Replacing in (3.2) the variable $t$ by $X$ and taking the expectation, we get $s^{2}-s^{4} \geq \mathbb{E} X^{3}$. Dividing by $s^{3}$ and using $\frac{\mathbb{E} X^{3}}{s^{3}} \geq g$, we derive $\frac{1}{s}-s \geq g$. Elementary considerations show that the latter inequality implies (3.1).

Lemma 3.2. Assume that a random variable $X \leq 1$ has mean $\mathbb{E} X=0$, variance $s^{2}=\mathbb{E} X^{2}$, and kurtosis such that $\frac{\mathbb{E} X^{4}}{s^{4}} \leq c$ with some $c \geq 1$. Then

$$
s^{2} \leq v(c), \quad 2 v(t) \stackrel{\text { def }}{=} t+1+\sqrt{(t+1)^{2}-4} .
$$

Proof. By Hölder's inequality we have $\frac{\mathbb{E} X^{4}}{s^{4}} \geq 1$. Hence, the condition $c \geq 1$ is natural. The function $v$ satisfies $v(c) \geq 1$ for $c \geq 1$. Therefore in cases where $s^{2} \leq 1$, inequality (3.3) turns to the trivial $s^{2} \leq 1 \leq v(c)$. Excluding this trivial case from the further considerations, we assume that $s^{2}>1$. Write $a=2 \sigma^{2}-1$. Then $a \geq 1$. It is clear that

$$
\left(t+s^{2}\right)^{2}(1-t)(a-t) \geq 0 \text { for } t \leq 1 .
$$

Replacing in (3.4) the variable $t$ by $X$ and taking the expectation, we get $\mathbb{E} X^{4} \geq s^{2}-s^{4}+s^{6}$. Dividing by $s^{4}$ and using $\frac{\mathbb{E} X^{4}}{s^{4}} \leq c$, we derive $\frac{1}{s^{2}}-1+s^{2} \leq c$. Elementary considerations show that the latter inequality implies (3.3).

Proof of Theorem 1.4. The proof starts with an application of the Chebyshev inequality similar to (1.7). This reduces the estimation of $\mathbb{P}\left\{M_{n} \geq x\right\}$ to estimation of expectations

$$
\mathbb{E} \exp \left\{h M_{n}\right\}, \quad \mathbb{E}\left(M_{n}-h\right)_{+}^{2} .
$$

As it is noted in the proof of Lemma 4.4 in B 2004, it suffices to estimate $\mathbb{E}\left(M_{n}-h\right)_{+}^{2}$ since the desired bound for the other expectation is implied by

$$
\mathbb{E}\left(M_{n}-h\right)_{+}^{2} \leq \mathbb{E}\left(T_{n}-h\right)_{+}^{2} .
$$

Let us prove (3.5). By Lemma 3.1 the condition $g_{k} \geq \gamma_{k}$ implies $s^{2} \leq u^{2}\left(\gamma_{k}\right)$. While applying Lemma 3.1 one has to replace $X$ by $X_{k}$, etc. In a similar way, by Lemma 3.2 the condition $c_{k} \leq \varkappa_{k}$ implies $s_{k}^{2} \leq v\left(\varkappa_{k}\right)$. Combining the inequalities and the assumption $s_{k}^{2} \leq \sigma_{k}^{2}$, we have

$$
s_{k}^{2} \leq \min \left\{\sigma_{k}^{2}, u^{2}\left(\gamma_{k}\right), v\left(\varkappa_{k}\right)\right\} .
$$

The inequality (3.6) together with the condition of the theorem yields $s_{k}^{2} \leq \alpha_{k}^{2}$. As it is shown in the proof of Theorem 1.1 in B 2004, the latter inequality implies (3.5). 


\section{REFERENCES}

[B] Bentkus, V., On measure concentration for separately Lipschitz functions in product spaces, Israel. J. Math. 158 (2007), 1-17.

[B] Bentkus, V., On Hoeffding's inequalities, Ann. Probab. 32 (2004), no. 2, 1650-1673.

[B] Bentkus, V., An inequality for tail probabilities of martingales with differences bounded from one side 16 (2003), no. 1, 161-173.

[B] Bentkus, V., A remark on the inequalities of Bernstein, Prokhorov, Bennett, Hoeffding, and Talagrand, Lith. Math. J. 42 (2002), no. 3, 262-269.

[B] Bentkus, V., An inequality for tail probabilities of martingales with bounded differences, Lith. Math. J. 42 (2002), no. 3, 255-261.

[B] Bentkus, V., An inequality for large deviation probabilities of sums of bounded i.i.d. random variables, Lith. Math. J. 41 (2001), no. 2, 112-119.

[BGZ] Bentkus, V., Geuze, G.D.C., and van Zuijlen, M., Optimal Hoeffding-like inequalities under a symmetry assumption, Statistics 40 (2006), no. 2, 159-164.

[BGZ] Bentkus, V., Geuze, G.D.C., and van Zuijlen, M., Unimodality: The linear case, Report no. 0607 of Dept. of Math. Radboud University Nijmegen (2006), 1-11.

[BGZ] Bentkus, V., Geuze, G.D.C., and van Zuijlen, M., Unimodality: The general case, Report no. 0608 of Dept. of Math. Radboud University Nijmegen (2006), 1-24.

[BGPZ] Bentkus, V., Geuze, G.D.C., Pinenberg, M.G.F., and van Zuijlen, M., Unimodality: The symmetric case, Report no. 0612 of Dept. of Math. Radboud University Nijmegen (2006), 1-12.

[BKZ] Bentkus, V., N. Kalosha, and van Zuijlen, M., Confidence bounds for the mean in nonparametric multisample problems, Statist. Neerlandica 61 (2007), no. 2, 209-231.

[BKZ] Bentkus, V., N. Kalosha, and van Zuijlen, M., On domination of tail probabilities of (super)martingales: explicit bounds, Lith. Math. J. 46 (2006), no. 1, 1-43.

[BZ] Bentkus, V., and van Zuijlen, M., On conservative confidence intervals, Lith. Math. J. 43 (2003), no. 2, 141-160.

van de Geer, S. A., On Hoeffding's inequalities for dependent random variables, Empirical process techniques for dependent data, Birkhauser Boston, Boston, MA Contemp. Math., 234, Am. Math. Soc., Providence, RI, 2002, pp. 161-169.

Godbole, A., and Hitczenko, P., Beyond the method of bounded differences, Microsurveys in discrete probability (Princeton, NJ, 1997), Dimacs Ser Discrete Math. Theoret. Comput. Sci., Amer. Math. Soc., Providence, RI 41 (1998), 43-58.

Hoeffding, W., Probability inequalities for sums of bounded random variables, J. Am. Statist. Assoc. 58 (1963), 13-30.

Laib, N., Exponential-type inequalities for martingale difference sequences. Application to nonparametric regression estimation, Comm. Statist. Theory Methods 28 (1999), 1565-1576.

McDiarmid, C., On the method of bounded differences, Surveys in combinatorics, 1989 (Norwich 1989), London Math. Soc. Lecture Note Ser., vol. 141, 1989, pp. 148-188.

Perron, F., Extremal properties of sums of Bernoulli random variables, Statist. Probab. Lett. 62 (2003), 345-354.

Pinelis, I., Toward the best constant factor for the Rademacher-Gaussian tail comparison, ESAIM Probab. Stat. 11 (2007), 412-426.

Pinelis, I., Inequalities for sums of asymmetric random variables, with applications, Probab. Theory Related Fields 139 (2007), no. 3-4, 605-635.

Pinelis, I., On normal domination of (super)martingales, Electron. J. Prabab 11 (2006), no. 39, $1049-1070$.

Pinelis, I., Fractional sums and integrals of $r$-concave tails and applications to comparison probability inequalities, Advances in stochastic inequalities (Atlanta, GA, 1997), Contemp. Math., 234, Am. Math. Soc., Providence, RI, 1999, pp. 149-168.

Pinelis, I., Optimal tail comparison based on comparison of moments, High dimensional probability (Oberwolfach, 1996), Progr. Probab., 43, Birkhäuser, Basel,, 1998, pp. 297-314.

Talagrand, M., The missing factor in Hoeffding's inequalities, Ann. Inst. H. Poincaré Probab. Statist. 31 (1995), no. 4, 689-702.

Vilnius institute of mathematics and informatics, Akademijos 4, LT-08663 Vilnius

E-mail address: bentkus@ktl.mii.lt, ??????????? 